\title{
New type of SSUrDNA sequence was detected from both Plasmodium ovale curtisi and Plasmodium ovale wallikeri samples
}

Mei Li, Zhigui Xia ${ }^{*}$ and He Yan

\begin{abstract}
Background: Plasmodium ovale is relatively unfamiliar to Chinese staff engaged in malaria diagnosis. In 2013, dried blood spots of four unidentified but suspected ovale malaria samples were sent to the National Malaria Reference Laboratory (NMRL) for reconfirmation.

Methods: Partial and complete, small, subunit ribosomal DNA (SSU rDNA) sequences of four samples were obtained with PCR-cloning-sequencing method. Obtained sequences were analyzed by aligning with each other and with nine SSU rDNA sequences of six known Plasmodium parasites. A phylogenetic tree was constructed based on complete SSU rDNA sequences and 12 same gene sequences derived from six known Plasmodium parasites and three Babesia parasites. Primary structure of conservative and variable regions of variant sequences was determined also by comparing them with those of six known Plasmodium parasites. To confirm their existence in genome, they were redetected with primers matching their variable regions. PCR systems aimed to roughly detect any eukaryotes and prokaryotes respectively were also applied to search for other pathogens in one of four patients.
\end{abstract}

Results: Totally, 19 partial and 23 complete SSU rDNA sequences obtained from four samples. Except eight variant sequences, similarities among sequences from same DNA sample were in general high (more than 98\%). The phylogenetic analysis revealed that three cases were infected by $P$. ovale wallikeri and one by $P$. ovale curtisi. Four of the variant sequences which obtained from four samples relatively showed high similarities with each other (98.5\%-100\%). Identical variant sequences actually could be re-obtained from each DNA sample. Their primary structure of conservative and variable regions showed quite fit with that of six known Plasmodium parasites. The test for prokaryote pathogens showed negative and the tests for eukaryotes only found DNA sequences of Human and P. ovale parasites.

Conclusion: Both P. ovale wallikeri and P. ovale curtisi infections are present in imported malaria cases of China. New type of partial SSU rDNA sequence which assumed to express in a certain life stage of $P$. ovale was obtained from both $P$. ovale wallikeri and $P$. ovale curtisi samples. This discovery would supply information and clues to identify and understand $P$. ovale parasites more accurately.

\section{Background}

Plasmodium ovale was the last described malaria parasites of humans [1]. However, relatively little attention had been paid to it since that time, because of its commonness, mild symptoms, usually low parasitaemia and same treatment method with $P$. vivax [2]. With the application of

\footnotetext{
* Correspondence: nipdxzhg@163.com

National Institute of Parasitic Diseases, Chinese Centre for Diseases Control and Prevention, Key Laboratory of Parasite and Vector Biology, Ministry of Public Health, WHO Collaborating Centre for Malaria, Schistosomiasis and Filariasis, Shanghai 200025, People's Republic of China
}

molecular methods in malaria diagnosis, more understanding of it had been achieved. Based on DNA polymorphisms in small subunit ribosomal RNA (SSU rRNA) gene, $P$. ovale parasites were defined into two types, a classic type and a variant type [3-8]. Lately, they were further confirmed based on the characterization of cysteine protease gene, ookinete surface protein gene and cytochrome b gene. Recently, these two types were certified to occur globally and so were formally named Plasmodium ovale curtisi

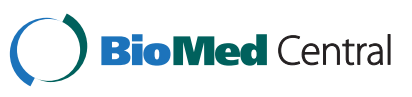

(c) 2014 Li et al.; licensee BioMed Central Ltd. This is an Open Access article distributed under the terms of the Creative Commons Attribution License (http://creativecommons.org/licenses/by/4.0), which permits unrestricted use, distribution, and reproduction in any medium, provided the original work is properly credited. The Creative Commons Public Domain Dedication waiver (http://creativecommons.org/publicdomain/zero/1.0/) applies to the data made available in this article unless otherwise stated. 
(typical type) and Plasmodium ovale wallikeri (variant type), respectively in honour of Christopher F Curtis (1993-2008) and David Walliker (1940-2007) [9].

Ovale malaria was commonly found in tropical West Africa and infrequently reported in Asia and Oceania [2,8-17]. In China, ovale malaria transmission and local infection was seldom reported except some occasionally imported cases. In a series of articles introduced the malaria situation in China during 2000-2012 [18-29], the ovale malaria situation was mentioned only since 2011 when the first 4 Provincial Reference Laboratories (PRLs) for malaria were established. Since that, 17 and 41 imported ovale malaria cases were confirmed respectively in laboratories in 2011 and 2012 [28,29]. So, $P$. ovale was relatively unfamiliar to Chinese staff engaged in malaria diagnosis, and $P$. ovale subspecies were even stranger for they are seldom differentiated in Chinese laboratories.

According to the requirement of China's National Malaria Elimination Programme (NMEP), every malaria case must be confirmed by the polymerase chain reaction (PCR) method. The nested-PCR system produced by Snounou in 1993 (NP-1993) [30] was recommended and widely adopted in PRLs. With the development of the reference system in China, staff began to be familiar with $P$. ovale. Unfortunately, microscopy positive but nested-PCR negative cases began to arise. In early 2013, dry blood spots of four unidentified but suspected ovale malaria patients were sent to the national malaria reference laboratory for reconfirmation.

Besides, stage-specific SSU rRNAs transcribed from structurally distinct nuclear genes had also been reported in some Plasmodium parasites, such as Plasmodium berghei, Plasmodium vivax and Plasmodium falciparum [31-35]. One type of these SSU rRNA genes is expressed in the asexual bloodstream and hepatic stage (A gene), and the other type is expressed in the mature parasites (sporozoites) of the mosquito ( $C$ gene or named $S$ gene in $P$. vivax) [31-33]. The third gene was $\mathrm{O}$ gene which was sampled and cloned from $P$. vivax and expressed in the maturing oocysts [34]. However, different stage-specific SSU rRNAs genes in $P$. ovale and Plasmodium malariae had not been reported.

\section{Methods}

\section{Ethical clearance}

The study was reviewed and approved by the Ethical Committee of National Institute of Parasite Diseases (NIPD), China Centre of Disease Control (CDC), and written informed consent was obtained from all the participants. No additional blood samples were taken from patients, other than those required for the primary diagnosis of malaria.

\section{Sample information and DNA sample preparation}

Dried blood spots of four malaria patients infected in Africa were supplied by Dalian CDC (labeled DL), Guizhou CDC (labeled GZ), Hainan CDC (labeled HN) and Shanghai CDC (labeled SH). The DL patient who was diagnosed in early 2012 had been to Equatorial Guinea. GZ, HN and SH patients who were diagnosed in late 2012 or early 2013 had been to Sierra Leone and Angola respectively. Genome DNA of them was prepared using QIAamp DNA Mini Kit (Quigen, 51306).

\section{Cloning and sequencing of SSU rDNA sequences of all samples}

All the samples were first reviewed with nest-PCR systems of NP-1993 [30]. The PCR products of the first round (partial SSU rDNA sequences) were cloned into pMDTM19-T Vector (Takara Vector Cloning Kit, 6013) and propagated in Escherichia coli DH5 cells under ampicillin selection. Positive plasmids were purified using Generay plasmid mini kit (Shanghai Generay Biotech Co Ltd, China, GK2004-100) and sequenced with Double DNA chain termination method (Shanghai MAP Biotech Co Ltd) on 3730XL DNA Analyzer. The plasmid DNA was assembled with software of DNA sequence assembly.

To confirm the results deduced from partial SSU rDNA sequences, primers to amplify complete SSU rDNA sequences were designed with software Oligo 6.0 based on complete SSU rDNA sequences of $P$. ovale wallikeri (Accession No. AB182491) download from National Center for Biotechnology Information (http://www.ncbi. nlm.nih.gov/). The amplified primers were UF: $5^{\prime}$-AACC TGGTTGATCTTGCCAGTAGTC-3' and RL: 5' -TAATG ATCCTTCCGCAGGTTC ACC-3'. The PCR, cloning and sequencing processes were the same as that used in obtained partial SSU rDNA sequences.

\section{Sequences analysis}

Assembled sequences amplified with same primers were aligned in software DNAman6.0. Conflicts among sequences were resolved by majority vote. Voted sequences were analyzed by aligning them with each other and with nine SSU rDNA sequences of six known Plasmodium parasites, $P$. falciparum (A and S type), P. vivax (A, S and O type), P. malariae, Plasmodium knowlesi, $P$. ovale curtisi, P. ovale wallikeri. GenBank accession numbers of nine SSU rDNA sequences were: M19172, M19173, U03079, U03080, U93235, M54897, AM910985, AB182491, AB182489. A phylogenetic tree was constructed based on complete SSU rDNA sequences and 12 same gene sequences from six known species and three species of Babesia parasites, Babesia rodhaini, Babesia.microti and Babesia divergens with software Mega 5.0, respectively. 
GenBank accession numbers of Babesia parasites were M87565, AB190459, AY046576.

The characteristics of variant SSU rDNA sequences were determined by comparing them with nine SSU rDNA sequences of known Plasmodium parasites with the help of software DNAman 6.0. The classification standard to determine the conservative or variable regions were quoted from Qari, 1994 [32]. Regions at which all sequences showed about $90 \%$ identity with each other were classified as conservative regions, $<60 \%$ as variable regions, and $60-90 \%$ as moderate similarity regions.

To confirm their presence in $P$. ovale genome, new primers, which matched the variable areas of variant sequence, were designed with software Oligo 6.0. They were shown in Table 1.

\section{Searching for other pathogens in $\mathrm{HN}$ patient}

Primers conserved for eukaryotes: NS1, 5'-GTAGTCAT ATGCTTGTCTC-3', NS2, 5'-GGCTGCTGGCACCAG ACTTGC-3' and for prokaryotes: 27F, 5' - AGA GTT TGA TCM TGG CTC AG-3', $(\mathrm{M}=\mathrm{C}$ or $\mathrm{A})$ and $1429 \mathrm{R}$, 5' - TAC GGY TAC CTT GTT ACG ACT T-3', $(\mathrm{Y}=\mathrm{C}$ or $\mathrm{T}$ ) were selected to search for other pathogens in $\mathrm{HN}$ patient. The PCR products which presented DNA bands on the gel was recovered and cloned into plasmid and sequenced.

\section{Results}

\section{Molecular diagnosing results with nest-PCR}

With NP-1993 system [30], except positive control samples of P. falciparum (205 bp) and P. vivax (120 bp), only DL sample showed $P$. ovale positive (800 bp) while the other three showed negative results (Figure 1). Therefore, DL patient should be infected by $P$. ovale.

\section{Characteristics of partial SSU rDNA sequences}

Nineteen sequences were obtained from four DNA samples. Eleven of them showed closest relationship with $P$. ovale wallikeri or $P$. ovale curtisi with high identities (more than 98\%). Eight of them showed the closest relationship with $P$. ovale wallikeri but with relatively low identities (79\%-92\%).

Four assembled sequences (numbered from pDL1-pDL4) of 1,069 bp-1,087 bp belonging to the DL sample were rigorously elected from 12 positive clones. Identities among them varied from 75.8 to $99.6 \%$. Sequences of pDL1, pDL2 and pDL3 showed closest relationship with $P$. ovale curtisi with identities of more than $98 \%$. But pDL4 showed the closest relationship to $P$. ovale wallikeri with identity of $79 \%$.

Three assembled sequences (numbered from pGZ1pGZ3) of 1,085 bp-1,090 bp belonging to GZ sample were rigorously elected from 20 positive clones. Identities among them varied from 89.1 to $95.9 \%$. All of them showed closest relationship with $P$. ovale wallikeri with relatively low identities of $86 \%-90 \%$.

Seven assembled sequences (numbered from pHN1pHN7) of 1,061 bp-1,091 bp belonging to the HN sample were rigorously elected from 23 positive clones obtained from four batches of reaction products. Identities among them varied from 79.2 to $99.4 \%$. Among them, the identities (91\%, 85\% and 92\%) of sequences pHN1, pHN3 and pHN5 with that of $P$. ovale wallikeri were much lower than others (more than 98\%).

Five assembled sequences (numbered from pSH1-pSH5) of 1,062 bp-1,091 bp belonging to the $\mathrm{SH}$ sample were rigorously elected from ten positive clones of $\mathrm{SH}$ sample. Identities among them varied from 74.3 to $99.8 \%$. But pSH3 showed an identity of $85 \%$ with SSU rDNA sequence of $P$. ovale wallikeri while the other sequences showed more than $98.5 \%$.

\section{Characteristics of complete SSU rDNA sequences}

In total, 23 complete SSU rDNA sequences were obtained from four DNA samples. They all showed closest relationship with $P$. ovale wallikeri or $P$. ovale curtisi with high identities (more than 98\%) but low identities (74.4\%-90.3\%) with eight variant sequences obtained in this study. For DL sample, six different assembled sequences $(2,104 \mathrm{bp})$ were obtained from ten positive clones (cDL1-cDL6). The identities among themselves were $99.0-99.8 \%$. Except of pDL4, identities between pDL and cDL sequences were between $98.3 \%$ and $99.7 \%$. No complete SSU rDNA sequences had identities with pDL4 were more than $78.2 \%$.

For GZ sample, seven different assembled sequences $(1,915$ bp-2,094 bp) of complete SSU rRNA gene were obtained from 18 positive clones and numbered

Table 1 Specific primers detecting variant SSU rDNA sequences in 4 DNA samples

\begin{tabular}{lll}
\hline Reaction turn & Primer sequences & Nucleotide position \\
\hline First round & Product size \\
PoWu & 5'-CGTTTCTGAGGTGCTACGCTTGG-3' & R2 (Low similarity) \\
PoWL1 & 5'-GACGACACTCGACTCGGTTATCC-3' & R7(Low similarity) \\
Second round & \\
PoWu & 5'-CGTTTCTGAGGTGCTACGCTTGG-3' & R2(Low similarity) \\
PoWL2 & 5'-GTATCTGATCGTCTTCACTCCCTT-3' & R4(High similarity) \\
\hline
\end{tabular}




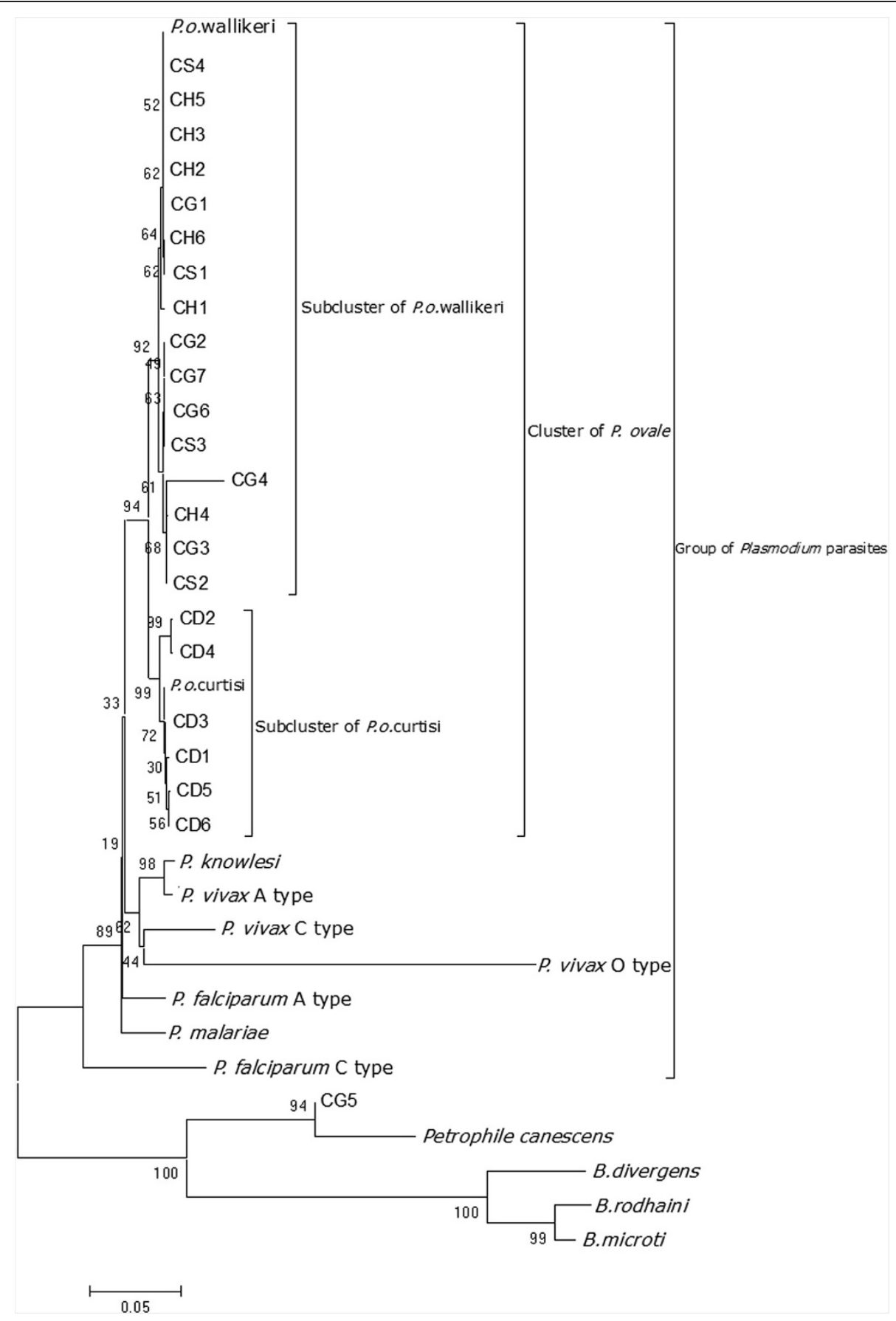

Figure 1 Phylogenetic tree representing the relationship of complete SSU rDNA sequences from four cases to another 13 taxa. CDL1-cDL6: 4 complete SSU rDNA sequences from Dailian sample, Genbank accession No.s were KF696369-KF696375; CHN1-cHN6: 6 complete SSU rDNA sequences from Hainnan sample, Genbank accession No.s were KF018654-KF018659; cGZ1-cGZ7: 7 complete SSU rDNA sequences from Guizhou sample, Genbank accession No.s were KF696359-KF696365; cSH1-cSH4: 4 complete SSU rDNA sequences from Shanghai sample, Genbank accession No.s were KF219558-KF219561.

cGZ1-cGZ7. The identities among themselves were between 69.7-99.8\%. Most variations presented in sequences of CG4 and CG5 which only showed about 90 and $81 \%$ identities with other sequences. There were rich variations among obtained cGZ sequences and pGZ sequences (with identities of 70.3\%-90.3\%).

For the HN sample, six different assembled sequences (2,091 bp-2,096 bp) were obtained from ten positive clones 
(CH1-CH6). The identities among themselves varied between 98.55-99.9\%. Except of pHN1, pHN3 and $\mathrm{pHN} 5$, identities between all $\mathrm{pHN}$ sequences and $\mathrm{cHN}$ sequences were between 98.0\%-99.4\%. No complete SSU rDNA sequences whose identities with pHN1, pHN3 and pHN5 were more than $90 \%$.

For SH sample, four assembled sequences (2,092 bp2,094 bp) of complete SSU rRNA gene were obtained from eight positive clones (cSH1-cSH4). The identities among them were between 98.4 to $99.6 \%$. Except of $\mathrm{pSH} 3$, the identities between all $\mathrm{pSH}$ sequences and cSH sequences were more than 98.1\% (98.1\%- 99.7\%). The identity of PS3 with complete SSU rDNA sequences was between 74.4 and $75.0 \%$.

\section{Phylogenetic analysis of complete SSU rDNA sequences}

The bootstrap consensus neighbouring-joining (NJ) tree (Figure 1) showed that most of the complete SSU rDNA sequences obtained in this study gathered in the cluster of $P$. ovale. Among them, most of the sequences from $\mathrm{HN}, \mathrm{GZ}$ and $\mathrm{SH}$ samples gathered in the subcluster in which $P$. ovale wallikeri was included, with bootstrap of $92 \%$. But no distinctively phylogenetic relationship presented between SSU rDNA sequences from three different samples. These results strongly supported all three cases being infections with P. ovale wallikeri. All the sequences obtained from DL sample made up the $P$. ovale cluster with $P$. ovale curtisi (bootstrap $=99 \%$ ). The sequence cGZ5 and the shrub stood out of Plasmodium parasites but together with Babesia parasites. The phyogenetic results were concordant with the reference deduced from sequences aligning results that $\mathrm{HN}, \mathrm{GZ}$ and $\mathrm{SH}$ showed closest relationship with $P$. ovale wallikeri, but DL with $P$. ovale curtisi.

\section{Redetection of new variant SSU rDNA sequences}

With the new nested PCR system, DNA fragments of aimed size of $440 \mathrm{bp}$ were present on the gel picture of all PCR products of four DNA samples. And their sequencing results of the PCR products showed that they were identical with pGZ1, PS3, PH3 and pDL4 respectively. However, no DNA bands present on the gel picture of the first round PCR products. This indicated that variant sequences truly existed in $P$. ovale. However, their copies in genome might be not as much as shown in positive clones.

\section{Characteristics of variant SSU rDNA sequences}

Summarily, among eight variant sequences, pGZ1 and pHN1 were identical with each other and showed 99.9\% identities with pSH3. These three sequences were all derived from $P$. ovale wallikeri samples and showed $97.9 \%$ identities with pDL4, which derived from $P$. ovale curtisi sample. Besides, two sequences from different samples,
pGZ2 and pHN5 were showed high similarity of 99.4\% with each other too. Similarities between variant sequences and known $P$. ovale SSU rDNA sequences were all less than 92\% as described previously. Same situation also presented between different Plasmodium species and different SSU rDNA types of same species.

The frequency of amplification and cloning of each the 8 variant SSU rDNA sequences among all sequences obtained from that isolate was calculated and is shown in Table 2. The four similar variant SSU rDNA sequences, pDL4, pGZ1, pHN3, and pSH3, showed relatively high percentage than other variant sequences (33-90\%). Among four of them, pDL4 showed the lowest amplified rate, which was 33\%, and pGZ1 showed the highest applied rate, which was $90 \%$. Each of the other four variant sequences, pGZ2, pGZ3, pHN1, and pHN5 only presented once.

Based on the alignment results, the primary structure of variant sequences was determined according to varying similarity of different regions between 10 DNA sequences of $P$. ovale (Additional file 1 ). They were divided into eight regions (R1-R8), four high similarity regions, two low similarity regions and two moderate similarity regions (Additional file 1 and Table 3). At R1, R4, R6 and R8, all variant sequences were showed high similarity with each other and those of $P$. ovale wallikeri (PoW) and P. ovale curtisi (PoC) (93\%-100\%). So these regions were high similarity or conservative regions. At R2 and R7, similarity of 39\%-54\% presented among most of the aligned sequences sourced from $P$. ovale. So, they were low similarity or variable regions. At R3 and R5, the similarity among them arranged within $62 \%-81 \%$. So they were moderate similarity regions.

Comparing the primary structure of variant sequences with that of nine known SSU rDNA sequences of Plasmodium species indicated that the division of R1-R8 could completely fit with that of Block5 - Block12 of complete Plasmodium SSU rRNA sequences displayed by Qari in 1994 (Additional file 1 and Table 3). Though similarities between DNA sequences of P.ovale were little more than those between different known Plasmodium species, the arrangement and positions of conservative, variable and moderate similarity regions of them were completely same.

Beside of the same structure of variant sequences with known Plasmodium parasites, the other interesting point was found. As shown in Additional file 1, the similarities of variant sequences with PoW or PoC varied greatly at different variable regions. Sequences pDL4, pGZ1, pHN3 and $\mathrm{pSH} 3$ showed high variable from PoW or PoC at all of four variable regions. But the other 4 sequences, pGZ2, pGZ3, pHN1 and pHN5 showed high variable from Pow only in some variable regions. Specifically, pHN1 showed nearly identical with PoW at R2 and R3 
Table 2 The occurred percentage of eight variant SSU rDNA sequences among the positive sequence clones

\begin{tabular}{lllllll}
\hline Name of the sequences & pDL4 & pGZ1 & pGZ2 + pGZ3 & pHN3 & pHN1 + pHN5 & pSH3 \\
\hline Percentage in positive clones & $33 \%(4 / 12)$ & $90 \%(18 / 20)$ & $10 \%(2 / 20)$ & $35 \%(8 / 23)$ & $9 \%(2 / 23)$ & $50 \%(5 / 10)$ \\
\hline
\end{tabular}

regions, pGZ2 at R7, pGZ3 at R5 and R7, and pHN5 at R7. At the other regions, they showed nearly identical with pGZ1 but high variations from Pow (Table 4).

Additionally, these four variable regions had selected as the targeted sites, designing primers to distinguish different Plasmodium species. For example, the R2 and R7 were the targeted sites of the ovale species-specific primers of rOVA1 and rOVA2 matched to [30].

\section{No other pathogen found in HN patient}

The PCR results with prokaryote primers showed negative while that with eukaryote primers showed positive. Sequencing results of six positive clones recovered from the main band turned out to be SSU rDNA of $P$. ovale parasites and the other six from the smears belonged to human DNA sequences.

\section{Discussion}

Malaria diagnosis was one of key steps of malaria control or elimination. Usually, three methods, that is microscopy, RDT and PCR, are used to detect malaria parasites. Unfortunately, the low and decreasing morbidity of malaria cases brought huge challenges to microscopists. Thus, clinicians or public health workers relied on RDT or PCR in malaria diagnosis more and more. However, quite a few RDT products were insensitive to some $P$. ovale. Besides, $P$. ovale often co-infected patients with P. falciparum or other Plasmodium parasites [5,10-12,16,36] and sometimes with a very low parasitaemia. So, if the PCR method could not find them out in time too, ovale patients might be delayed or neglect treatment. Then ovale malaria transmission or relapse might be resulted from the parasites lingered in patients' livers $[15,37,38]$ which would threat the populations seriously.

SSU rRNA gene was the most widely accepted molecular marker in identifying different malaria parasites infecting humans with PCR method. The common PCR systems based on SSU rDNA sequences were NP-1993 [30]. Latterly, NP-2002 [39] and NP-2005 [36] were developed as new variant SSU rDNA sequences were discovered. At first, the P. ovale wallikeri made the NP-1993 system unreliable [7,36] and the system of NP-2002 seemed to resolve this problem, in which not only the PCR product size of first round extended from 1,100 $\mathrm{bp}$ to $1,700 \mathrm{bp}$ along the forward direction of SSU rDNA sequences, but the reverse ovale-specific primer was designed at conserved region. However, another PCR system of NP-2005 in which a pair of new P. ovale species-specific primers was applied, proved to be more accurate than both NP-1993 and NP-2002 in detecting P. ovale. For the present, the problem is that one of the species-specific primers used in these three systems is located at the variable region $\mathrm{R} 2$ or $\mathrm{B} 6$. In this region, variant sequences obtained in this study showed low similarity $(<60 \%)$ with normal ones. But high similarity (>90\%) presented between variant and normal ones where genus-specific primers usually matched. So, theoretically, all the SSU rDNA types could be amplified synchronously with same genus-specific primers. If most of the PCR products obtained in the first round reaction did not match the species-specific primer (as shown in this study), all of the PCR results in the three systems

Table 3 Comparison of primary structure of conservative and variable regions between variant sequences and other Plasmodium parasites*1

\begin{tabular}{|c|c|c|c|c|c|c|c|c|}
\hline \multicolumn{2}{|c|}{ Block no } & \multicolumn{2}{|c|}{ Nucleotide position } & \multicolumn{2}{|l|}{ Similarity } & \multicolumn{3}{|l|}{$(\%)$} \\
\hline $\mathrm{PVC}$ & $\mathrm{pGZ1}$ & $\mathrm{PVC}$ & $\mathrm{pGZ1}$ & VPVC & pGZ1 & PVC & $\mathrm{pGZ1-1{ } ^ { * 3 }}$ & $p G Z 1-2^{* 4}$ \\
\hline B5 & R1 & $54-656$ & $1-44^{* 2}$ & High & High & 95 & 100 & 100 \\
\hline B6 & $\mathrm{R} 2$ & $657-804$ & $45-207$ & Low & Low & 26 & $39-40$ & 36 \\
\hline B7 & R3 & $805-927$ & 208-333 & Moderate & Moderate & 74 & $78-81$ & 76 \\
\hline B8 & R4 & $928-1,112$ & $334-517$ & High & High & 92 & 93 & 93 \\
\hline B9 & R5 & $1,113-1,194$ & $575-603$ & Low & Low-Moderate & 49 & $62-65$ & 52 \\
\hline $\mathrm{B} 10$ & R6 & $1,195-1,468$ & $605-876$ & High & High & 97 & 97 & 97 \\
\hline B11 & R7 & $1,467-1,687$ & $878-1,033$ & Low & Low & 34 & $53-54$ & 40 \\
\hline B12 & $\mathrm{R} 8$ & 1,688-1,793 & $1,034-1,090$ & High & High & 94 & 93-95 & 95 \\
\hline
\end{tabular}

${ }^{* 1}$ Data of the other Plasmodium parasites and classification standard were quoted from Qari [32].

$*^{2}$ One nucleotide base (A) on sequence of $P$. vivax C type (PVC) (Nucleotide position 656) deleted on PG1.

${ }^{*}$ Similarity between $\mathrm{PG} 1$ and corresponding sequences of $P$. ovale wallikeri and $P$. ovale curtisi.

${ }^{*}$ Similarity between PG1 and corresponding sequences of $P$. vivax $C$ type. 
Table 4 Detail distributions of 4 variable regions in 8 variant SSU rDNA sequences

\begin{tabular}{lllll}
\hline & R2 & R3 & R5 & R7 \\
\hline pDL4 & $x$ & $x$ & $x$ & $x$ \\
pGZ1 & $x$ & $x$ & $x$ & $x$ \\
pGZ2 & $x$ & $x$ & $x$ & $/$ \\
pGZ3 & $x$ & $x$ & $/$ & $/$ \\
pHN1 & $/$ & $/$ & $x$ & $x$ \\
pHN3 & $x$ & $x$ & $x$ & $x$ \\
pHN5 & $x$ & $x$ & $x$ & $/$ \\
pSH3 & $x$ & $x$ & $x$ & $x$ \\
\hline
\end{tabular}

might show negative. So, new system which could detect both $P$. ovale curtisi and $P$. ovale wallikeri was required. However, the glad thing for hospitals or CDCs which owned real-time quantitative PCR instruments was that some new assays developed in recent years might not be borrowed to apply. For example, in the assay created by Bauffe in 2012 [13], the forward primer POF, 5' -ATAAACTATGCCGACTAGGTT-3' matching to $P$. ovale curtisi and $P$. ovale wallikeri specifically covered both R4 and R5 and possessed 2 nts different from variant sequences, while the reverse primer POR, 5'ACTTTGATTTCTCATAAGGTACT-3' covered both R5 and $\mathrm{R} 6$ and possessed $1 \mathrm{nt}$ different from variant sequences. So the variant sequences could not bother it.

For the variant sequences, the possibility had been suspected that they might derive from the other pathogen which co-infected patients with $P$. ovale. But two excuses denied it. The first was that no other Plasmodium parasites were found with specific primers to complete SSU rDNA sequences and no other pathogens were detected with PCR systems specific to both prokaryote and eukaryote organisms. The second was that these four patients were infected by $P$. ovale parasites in different time and at different places, and furthermore they also lived faraway from each other in China. So the other assume was suggested that they might belong to one type of SSU rRNA gene which expressed in a certain life stage of $P$. ovale parasites as A, C and O types of SSU rRNA genes did in $P$. falciparum and $P$. vivax. As known, three SSU rRNA gene types were confirmed in $P$. vivax, two in $P$. falciparum and three in P. berghei [31-35]. However, only one type of SSU rDNA sequences was identified in each of the $P$. ovale species. Except of their same primary organization of conservative and variable regions, the little higher similarities (99.9\%-100\%) among variant sequences (pGZ1, pHN3 and pSH3), which derived from $P$. ovale wallikeri patients than that $(97.9 \%)$ between variant sequences derived from different $P$. ovale subspecies supported this assumption too. Otherwise, analogous sequences were found by other scientists. So it was confident to believe that the variant sequences belong to one type of SSU rDNA sequence of $P$. ovale. Whatever, true evidence such as analysis on transcript of $P$. ovale sporozoites or genome DNA of $P$. ovale is required to confirm this assume.

In the result of this study, there was a conflict in that no complete SSU rDNA sequences showed high similarity with the variant sequences. There may be two reasons: either the primers were not conserved enough or there were fewer copies of variant SSU rDNA than the normal ones. For the first excuse, the primers used to amplify complete SSU rDNA sequences were conserved in all the other five Plasmodium parasites, so the conflict unlikely resulted from it. Therefore, it seems that the different copies of them in the whole genome DNA had led to the results. Regrettably, no such research has mentioned it.

Except of pDL4, pGZ1, pHN3 and pSH3, there were other 4 special variant sequences, pGZ2, pGZ3, pHN1 and pHN5 were also detected. Their character showed high similarities with PoW in some variable regions and low similarities in the other variable regions. This result indicated that hybridization or recombination might have occurred between different types of SSU rRNA genes. But more studies were required to discover if they had really happened in the process of ovale parasites development or just were missing PCR products.

In a word, variant sequences obtained from $P$. ovale samples on the one hand might make some PCR systems invalid, but on the other hand, they supply information and clues to identify and understand $P$. ovale parasites more accurately.

\section{Additional file}

Additional file 1: Aligning results of variant sequences with SSU rDNA sequences of six known Plasmodium parasites and their primary structure of conservative and variable regions. Dots represent similarity with $P$. ovale wallikeri (PoW) and dashes represent gaps introduced to align the sequences. $P \vee A, P v O$ and $P \vee S$ represent $A, O$ and S SSU rDNA sequences of $P$. vivax; PfA and PfS represent $A$ and $S$ of $P$. falciparum; Pm, Pk and PoC represent $P$. malariae, $P$. knowlesi and $P$. ovale curtisi.

\section{Competing interests}

The authors declare that they have no competing interests.

\section{Authors' contributions}

ZGX carried out the revising manuscript draft and collected the samples and their epidemic information of four patients. HY carried out the DNA preparation and PCR diagnosis with NP-1993 method. ML finished the other work. All authors read and approved the final manuscript.

\section{Acknowledgements}

We would like to thank Hainan provincial CDC, Guizhou provincial CDC, Shanghai city CDC and Dalian city CDC who supplied samples to us. This study were supported by Quality Inspection of Public Welfare Scientific Research Project (No. 2012424056) and National Science and Technology Major Project (No. 2012ZX10004-220) 
Received: 14 March 2014 Accepted: 21 May 2014

Published: 3 June 2014

\section{References}

1. Stephens JWW: A new malaria parasite of man. Ann Trop Med Parasitol 1922, 16:383-388.

2. Collins WE, Jeffery GM: Plasmodium ovale: parasite and disease. Clin Microbiol Rev 2005, 18:570-581

3. Li J, Wirtz RZ, McConkey GA, Sattabongkot J, Waters AP, Rogers MJ, McCutchan TF: Plasmodium: genus-conserved primers for species identification and quantitation. Exp Parasitol 1995, 81:182-190.

4. Kimura M, Kaneko O, Liu Q, Zhou M, Kawamoto F, Wataya Y, Otani S, Yamaguchi $Y$, Tanabe $K$ : Identification of the four species of human malaria parasites by nested PCR that targets variant sequences in the small subunit rRNA gene. Parasitol Int 1997, 46:91-95.

5. Zhou M, Wongsrichanalai C, Wuwonkerd W, Panar K, Prajakwong S, Pensiri A, Kimura M, Matsuoka H, Ferrerira MU, Isomura S, Kawamoto F: High prevalence of Plasmodium malariae and Plasmodium ovale in malaria patients along the Thai-Myanmar border, as revealed by acridine orange staining and PCR-based diagnoses. Trop Med Int Health 1998, 3:304-312.

6. Kawamoto F, Liu Q, Ferreira MU, Tantular IS: How prevalent are Plasmodium ovale and P. malariae in East Asia? Parasitol Today 1999, 15:422-426.

7. Win TT, Jalloh A, Tantular IS, Tsuboi T, Ferreira MU, Kimur M, Kawamoto F: Molecular analysis of Plasmodium ovale variants. Emerg Infect Dis 2004, 10:1235-1240.

8. Tachibana M, Tsuboi T, Kaneko O, Khuntirat B, Torii M: Two types of Plasmodium ovale defined by SSU rRNA have distinct sequences for ookinete surface proteins. Mol Biochemi Parasitol 2002, 122:223-226.

9. Sutherland CJ, Tanomsing N, Nolder D, Oguike M, Jennison C, Pukrittayakamee S, Dolecek C, Hien TT, Do Rosário VE, Arez AP, Pinto J, Michon P, Escalante AA, Nosten F, Burke M, Lee R, Blaze M, Otto TD, BarnwellJ W, Pain A, Williams J, White NJ, Day NP, Snounou G, Lockhart PJ, Chiodini PL, Imwong M, Polley SD: Two non-recombining sympatric forms of the human malaria parasite Plasmodium ovale occur globally. J Infect Dis 2010, 201:1544-1550.

10. Win T, Kin K, Mizuno S, Zhou M, Liu Q, Ferreira MU, Tantular IS, Kojima S, Ishii A, Kawamoto F: Wide distribution of Plasmodium ovale in Myanmar. Trop Med Int Health 2002, 7:231-239.

11. Fuehrer HP, Habler VE, Fally MA, Harl J, Starzengruber P, Swoboda P, Bloeschl I, Khan WA, Noedl H: Plasmodium ovale in Bangladesh: Genetic diversity and the first known evidence of the sympatric distribution of Plasmodium ovale curtisi and Plasmodium ovale wallikeri in southern Asia. Intern J Parasitol 2012, 42:693-699.

12. Dinko B, Oguike MC, Larbi JA, Bousema T, Sutherland CJ: Persistent detection of Plasmodium falciparum, $P$. malariae, $P$. ovale curtisi and $P$. ovale wallikeri after ACT treatment of asymptomatic Ghanaian school-children? Intern J Parasitol Drugs Drug Resist 2013, 3:45-50.

13. Bauffe F, Desplans J, Fraisier C, Parzy D: Real-time PCR assay for discrimination of Plasmodium ovale curtisi and Plasmodium ovale wallikeri in the Ivory Coast and in the Comoros Islands. Malar J 2012, 11:307.

14. Oguike MC1, Betson M, Burke M, Nolder D, Stothard JR, Kleinschmidt I, Proietti C, Bousema T, Ndounga M, Tanabe K, Ntege E, Culleton R, Sutherland CJ: Plasmodium ovale curtisi and Plasmodium ovale wallikeri circulate simultaneously in African communities. Intern J Parasitol 2011 41:677-683

15. DeLaval F, Oliver M, Rapp C, De Santi VP, Mendibil A, Deparis X, Simon F: The challenge of diagnosing Plasmodium ovale malaria in travelers: report of six clustered cases in French soldiers returning from West Africa. Malar J 2010, 9:358.

16. Mueller I, Zimmerman PA, Reeder JC: Plasmodium malariae and Plasmodium ovale - the 'bashful' malaria parasites. Trends Parasitol 2007, 23:278-283

17. Su XZ: Human malaria parasites: are we ready for a new species? J Infect Dis 2010, 201(10):1453-1454.

18. Expert Advisory Committee on Malaria, Ministry of Health: Malaria situation in the People's Republic of China in 2000 (in Chinese). Chin J Parasitol Parasit Dis 2001, 19:257-259.

19. Gao CY, Chai GJ, Han GH, Yang XW, Liu L, Jiang ZJ: [The incidence trend of Malaria in the people's Republic of China during 1950-2001](in Chinese). Chin J Public Health 2003, 19:725-726.
20. Zhou SS, Tang LH, Sheng HF: [Malaria situation in the People's Republic of China in 2002](in Chinese). Chin J Parasitol Parasit Dis 2005, 23:385-387.

21. Zhou SS, Tang LH, Sheng HF, Wang Y: [Malaria situation in the People's Republic of China in 2004](in Chinese). Chin J Parasitol Parasit Dis 2006, $24: 1-3$.

22. Zhou SS, Wang Y, Tang LH: [Malaria situation in the People's Republic of China in 2005](in Chinese). Chin J Parasitol Parasit Dis 2006, 24:401-403.

23. Zhou SS, Wang Y, Tang LH: [Malaria situation in the People's Republic of China in 2006](in Chinese). Chin J Parasitol Parasit Dis 2007, 25:439-441.

24. Zhou SS, Wang Y, Fang W, Tang LH: [Malaria situation in the People's Republic of China in 2007. Chin J Parasitol Parasit Dis 2008, 26:401-403.

25. Zhou SS, Wang Y, Fang W, Tang LH: [Malaria situation in the People's Republic of China in 2008](in Chinese). Chin J Parasitol Parasit Dis 2009, 27:455-457.

26. Zhou SS, Wang Y, Xia ZG: [Malaria situation in the People's Republic of China in 2009](in Chinese). Chin J Parasitol Parasit Dis 2011, 29:1-3.

27. Zhou SS, Wang Y, Li Y: [Malaria situation in the People's Republic of China in 2010](in Chinese). Chin J Parasitol Parasit Dis 2011, 29:401-403.

28. Xia ZG, Yang MN, Zhou SS: [Malaria situation in the People's Republic of China in 2011](in Chinese). Chin J Parasitol Parasit Dis 2012, 30:419-422.

29. Xia ZG, Feng J, Zhou SS: [Malaria situation in the People's Republic of China in 2012](in Chinese). Chin J Parasitol Parasit Dis 2013, 30:413-418.

30. Snounou G, Viriyakosol S, Zhu XP, Jarra W, Pinheiro L, do Rosa-rio VE, Thaithong S, Brown KN: High sensitivity of detection of human malaria parasites by the use of nested polymerase chain reaction. Mol Biochem Parasitol 1993, 61:315-320.

31. Gunderson JH, Sogin ML, Wollett G, Hollingdale M, De La Cruz VF, Waters AP, McCutchan TF: Structurally distinct, stage-specific ribosomes occur in Plasmodium. Science 1987, 238:933-937.

32. Qari SH, Goldman IF, Pieniazek NJ, Collins WE, Lal AA: Blood and sporozoite stage-specific small subunit ribosomal RNA-encoding genes of the human malaria parasite Plasmodium vivax. Gene 1994, 150:43-49.

33. McCutchan TF, De La Cruz VF, Lal AA, Gunderson JH, Elwood HJ, Sogin ML: The primary sequence of two small subunit ribosomal genes from Plasmodium falciparum. Mol Biochem Parasitol 1988, 28:63-68.

34. Li J, Gutell RR, Damberger SH, Wirtz RA, Kissinger JC, Rogers MJ, Sattabongkot J, McCutchan TF: Regulation and trafficking of three distinct $18 \mathrm{~S}$ ribosomal RNAs during development of the malaria parasite. J Mol Biol 1997, 269:203-213.

35. Li J, McConkey GA, Rogers MJ, Waters AP, McCutchan TR: Plasmodium: The developmentally regulated ribosome. Exp Parasitol 1994, 78:437-441.

36. Calderaro A, Piccolo G, Perandin F, Gorrini C, Peruzzi S, Zuelli C, Ricci L, Manca N, Dettori G, Chezzi C, Snonou G: Genetic polymorphisms influence Plasmodium ovale PCR detection accuracy. J Clin Microbiol 2007, 45:1624.

37. Tordrup D, Virenfeldt J, Andersen FF, Petersen E: Variant Plasmodium ovale sampled from a patient infected in Ghana. Malar J 2011, 10:15.

38. Kang YJ, Yang JY: A case of Plasmodium ovale malaria imported from West Africa. Korean J Parasitol 2013, 51:213-218.

39. Snounou $G$, Singh B: Nested PCR analysis of Plasmodium parasites. Meth Mol Med 2002, 72:189-203.

doi:10.1186/1475-2875-13-216

Cite this article as: Li et al.: New type of SSUrDNA sequence was detected from both Plasmodium ovale curtisi and Plasmodium ovale wallikeri samples. Malaria Journal 2014 13:216.

\section{Submit your next manuscript to BioMed Central and take full advantage of:}

- Convenient online submission

- Thorough peer review

- No space constraints or color figure charges

- Immediate publication on acceptance

- Inclusion in PubMed, CAS, Scopus and Google Scholar

- Research which is freely available for redistribution 